\title{
Evaluation of provincial initiatives in palliative care: a baseline assessment of pre-implementation patient perspectives 50
}

\section{a ge ncy or proviatheath services athority}

\section{Background}

- Early advanced care planning (ACP) conversations show improved patient outcomes. However, content and timing of these end of life discussions have been variable.

- Based on a recent needs assessment from renal care providers, the BC Integrated Palliative Nephrology Working Group has implemented a multipronged approach to improve palliative care in nephrology.

- This large-scale project requires systematic evaluation to demonstrate its efficacy

\section{Objectives}

To assess baseline experiences and perspectives on ACP in patients with advanced renal disease across 5 Health Authorities (HAs) in BC.

Methods

- Pre-implementation telephone interviews $(\sim 15$ minutes each) were conducted with 30 randomly selected patients, 6 from each provincial HA (Table 1).

- The interview was designed based on a validated questionnaire (Figure 1) to audit ACP (Heyland et al. 2012).

Interviews were analyzed quantitatively for patients involvement in ACP and qualitatively for common themes.

- This assessment will be repeated in 1 year postimplementation to evaluate improvements to the palliative care approach.

\section{Outcomes}

- Majority of patients interviewed have advanced directives (Figure 2A); this is more common in patients on dialysis than in patients with CKD (Figure 2C).

- Only $30 \%$ of patients had detailed discussions with their health providers about life-sustaining therapy or palliation in case of a life-threatening condition; this varied across HAs.

- While most patients did not find ACP discussions to be difficult, they would prefer if providers initiated discussion at multiple time points and had more time to provide information.

Bibliography

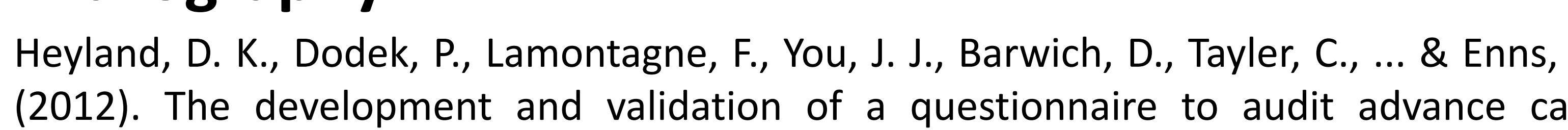
planning (ACP). BMI supportive \& palliative care, 2(2), 175-176.

Questions? Please contact:

Dr. Juliya Hemmett: Juliya.Hemmett@gmail.com
Alice Wang ${ }^{1}$, Sarah Thomas ${ }^{2}$, Adeera Levin ${ }^{1,2}$, Gaylene Hargrove ${ }^{2}$, Juliya Hemmett ${ }^{2}$. 1. University of British Columbia, 2. BC Renal Agency

Table 1. Patient Demographics

\begin{tabular}{lcccccc} 
& A & B & C & D & E & All Sites \\
\hline Age & 73.50 & 84.33 & 67.83 & 76.17 & 75.00 & 75.37 \\
Time since Dialysis initiation & 43.5 & 46.25 & 20 & 63.25 & 17.5 & 38.10 \\
(months) & & & & & & \\
Gender (\% male) & 66.67 & 50.00 & 50.00 & 66.67 & 66.67 & 60.00 \\
Race (\% Caucasian) & 100.00 & 50.00 & 100.00 & 83.33 & 100.00 & 86.67
\end{tabular}
Q1: Do you have an advanced care directive or living
will?

Q2A: Have you thought about whether you would/wouldn't want life-sustaining treatments in case your physical health deteriorated?

Q2B: Have you discussed your wishes with anyone?

Q5: Has a doctor/health professional discussed palliative/spiritual care that might be helpful if you had a palliative/spiritual care that
life-threatening condition?

Q6: Has a doctor or other member of health care team provided information about supportive care services such as palliative and spiritual that may be helpful if you had a life-threatening illness?

Q7: Has a doctor asked what is important to you as you consider health care decisions at this stage of your life? Q8: Has a doctor talked to you about the benefits and burdens of life sustaining medical treatments?

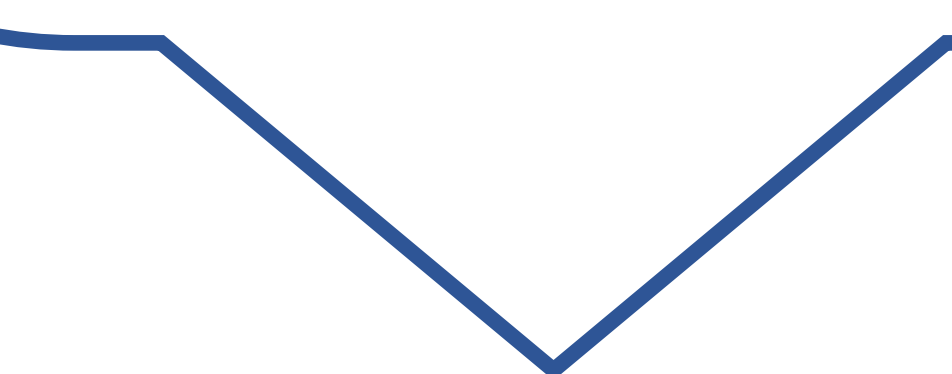

Figure 1. Selected Questions from Validated Questionnaire.

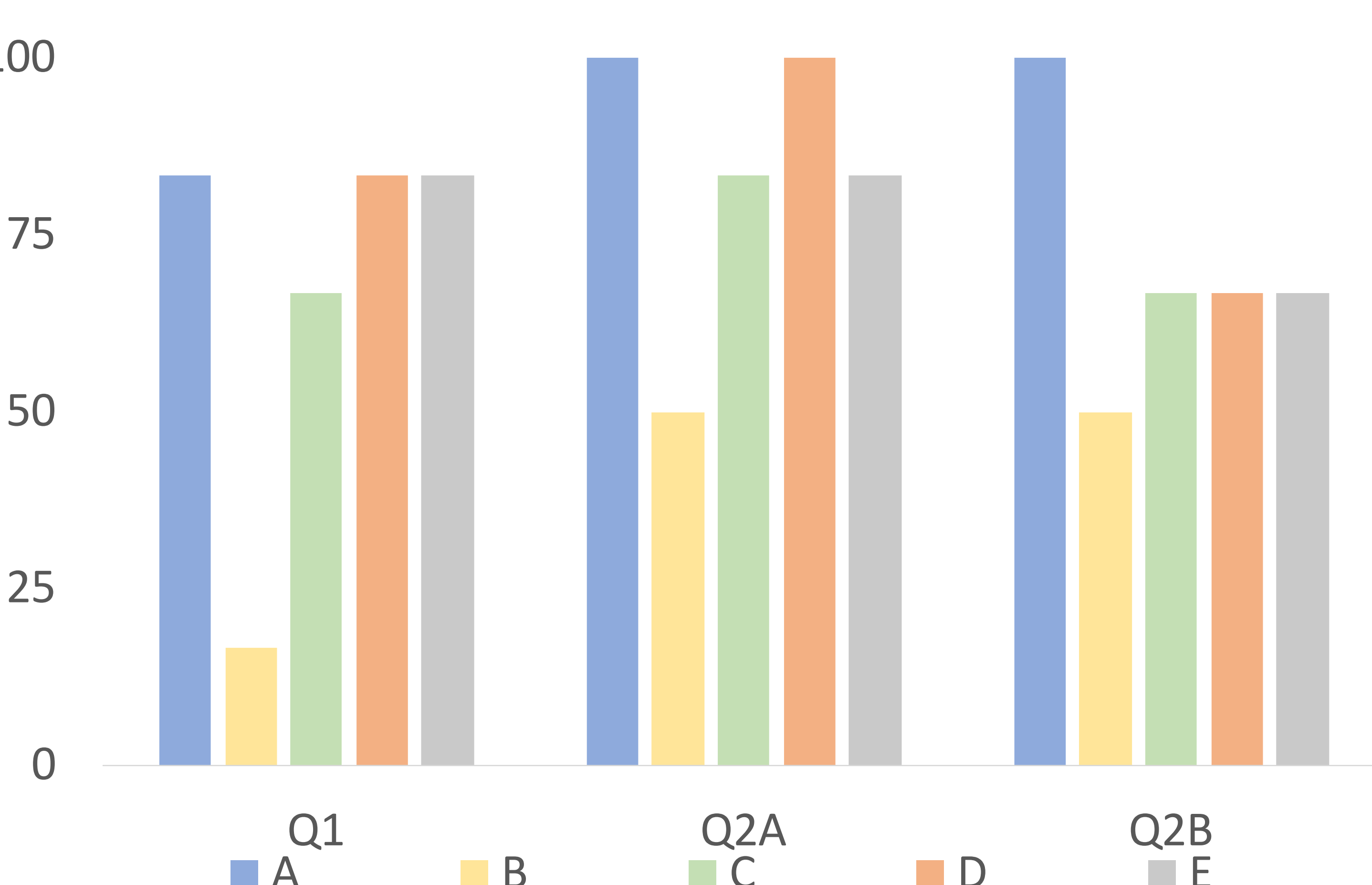

Figure 2B. \% of Patients who reported having advanced directives (Q1), considered treatment plans (Q2A), and had ACP discussions (Q2B) stratified by HAs A-E.

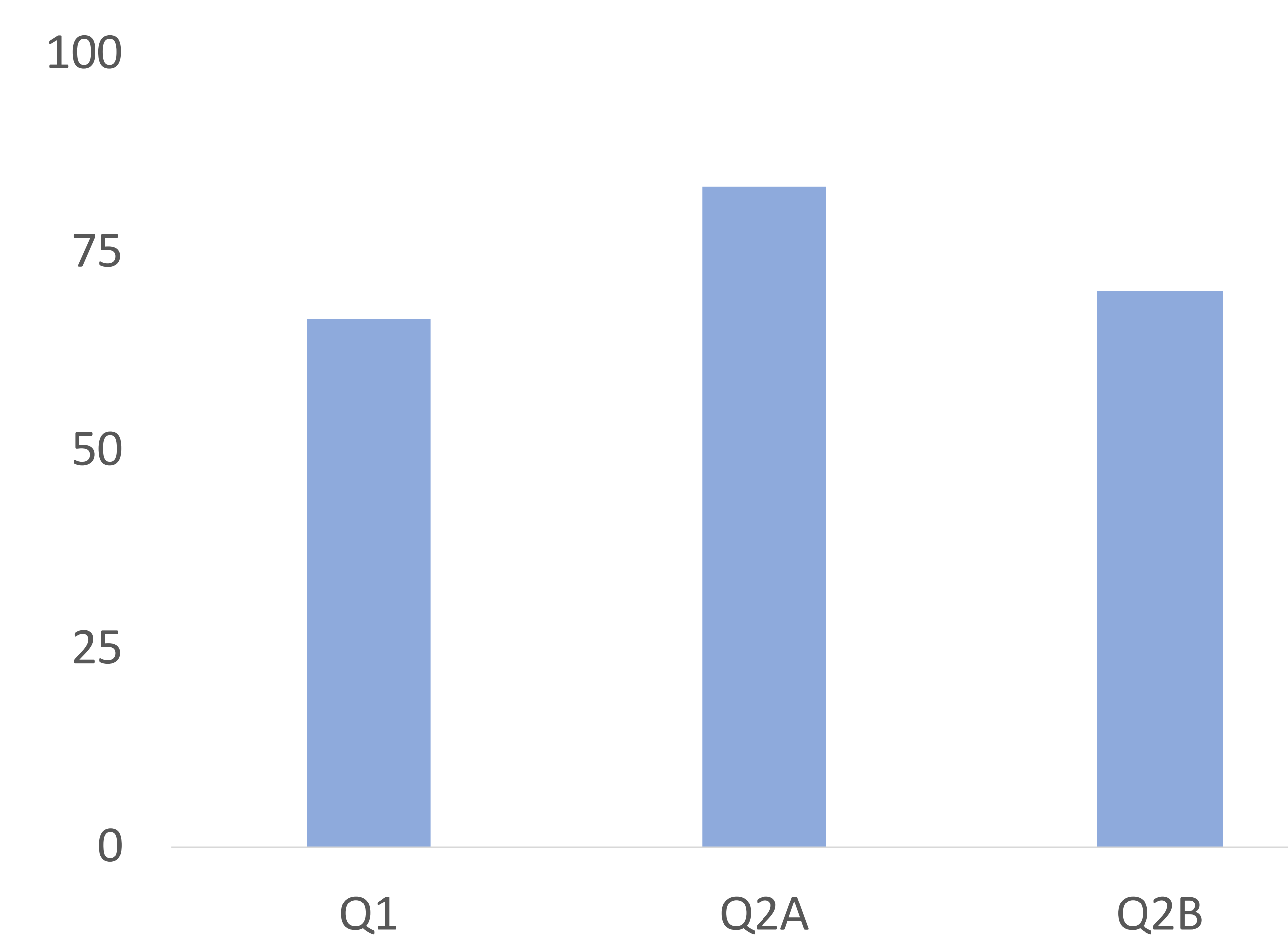

Figure 2A. \% of Patients who reported having advanced directives (Q1) considered treatment plans (Q2A), and had ACP Discussions (Q2B) across all HAs.

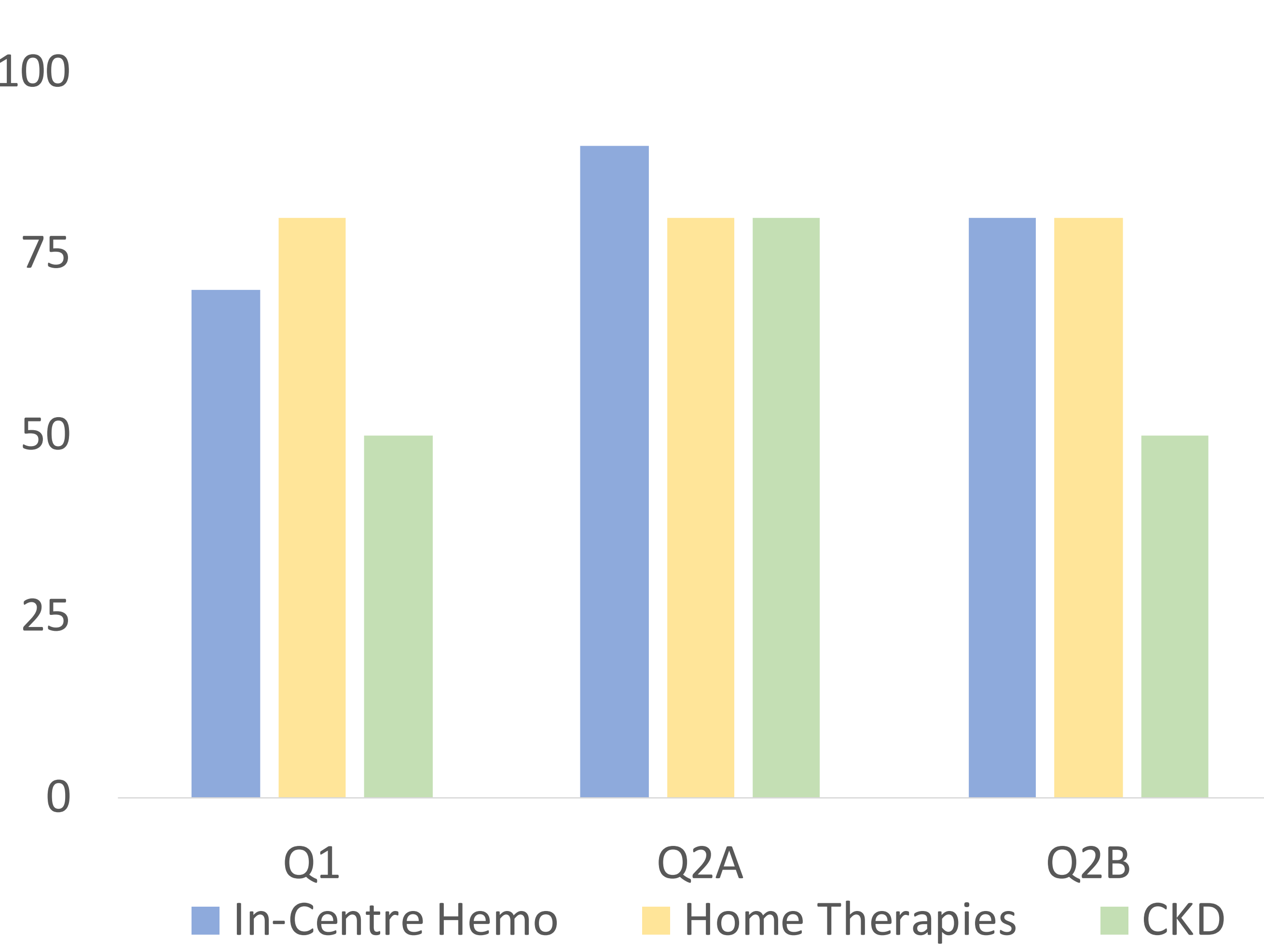

Figure 2C. \% of Patients who reported having advanced directives (Q1), considered treatment plans (Q2A), and had ACP discussions (Q2B) stratified by dialysis modality.

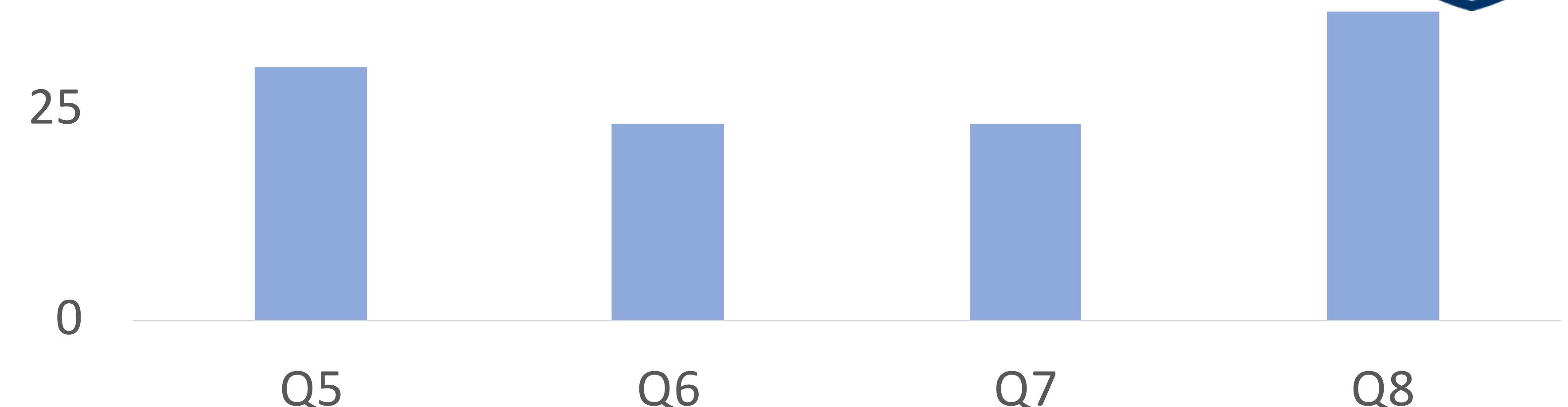

Figure $3 \mathrm{~A} . \%$ of patients reporting ACP discussions with doctors across all HAs. 50

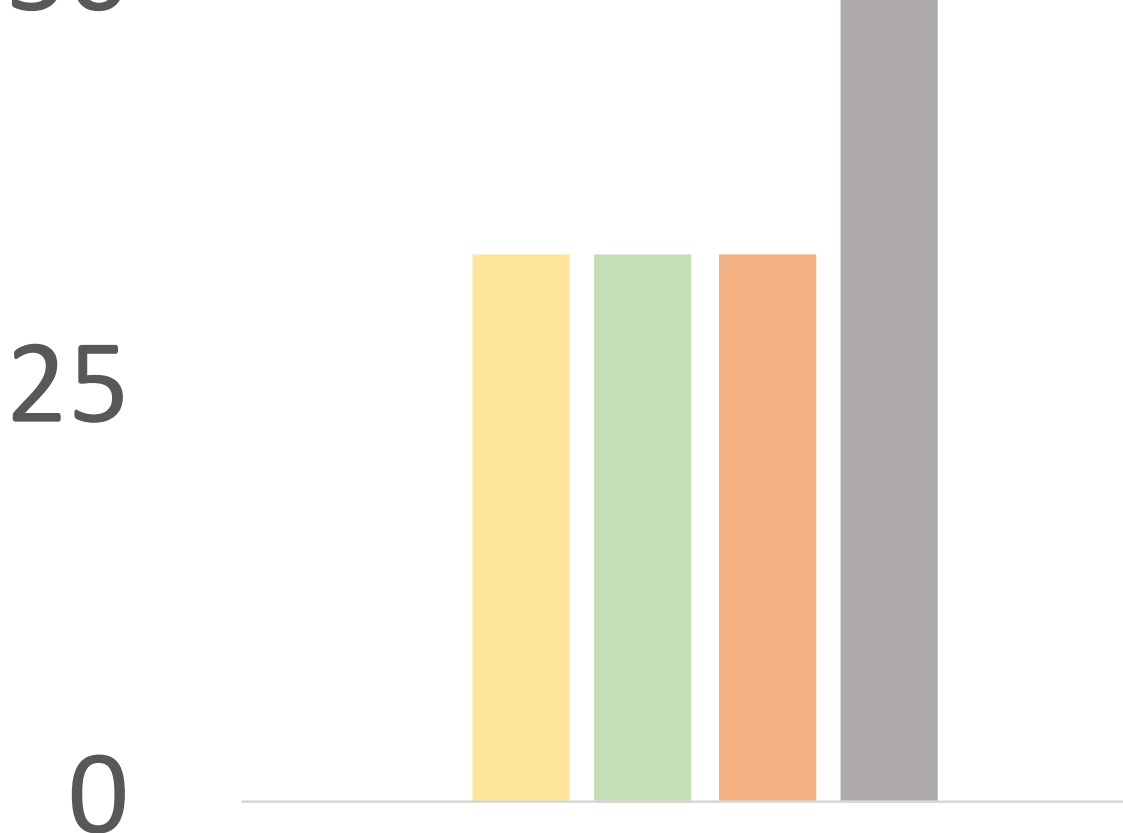

Q5

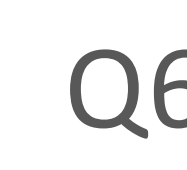

Q6

Q7

$A \square B \square C \backsim D \backsim E$

Figure 3B. \% of patients reporting ACP discussions with doctors stratified by HAs A-E.

50

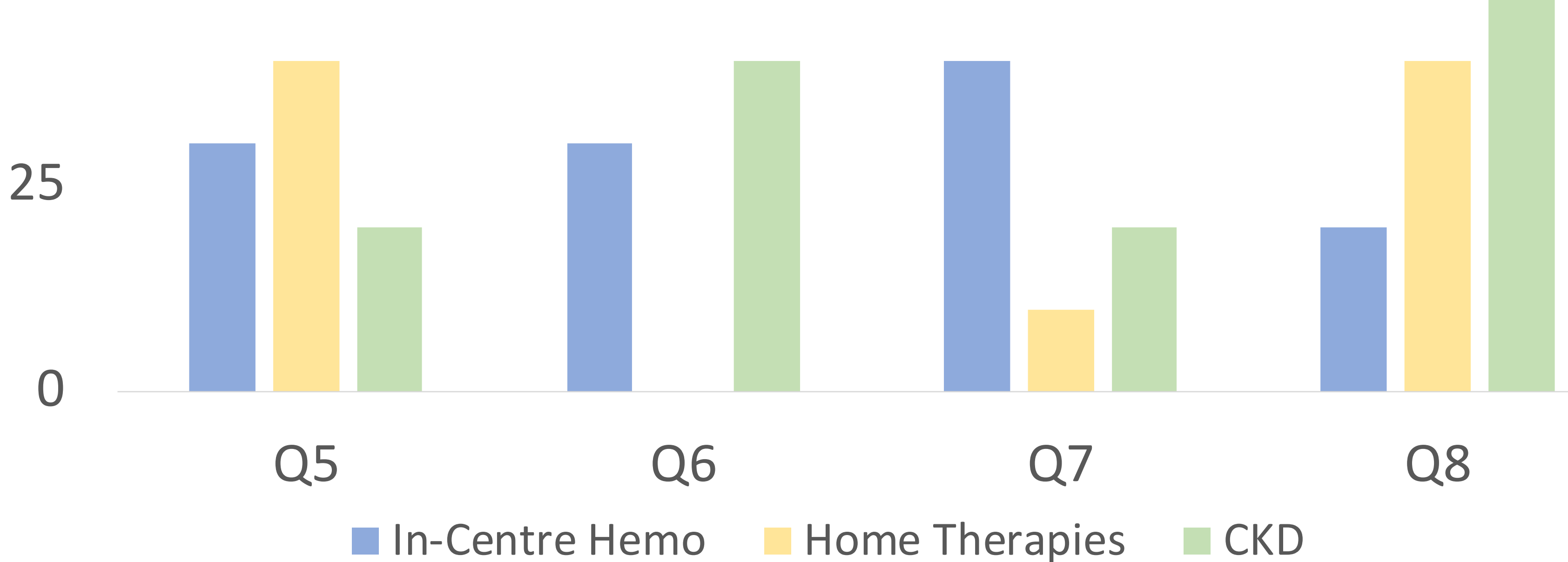

Figure 3C. \% of patients reporting ACP discussions with doctors stratified by dialysis modality.

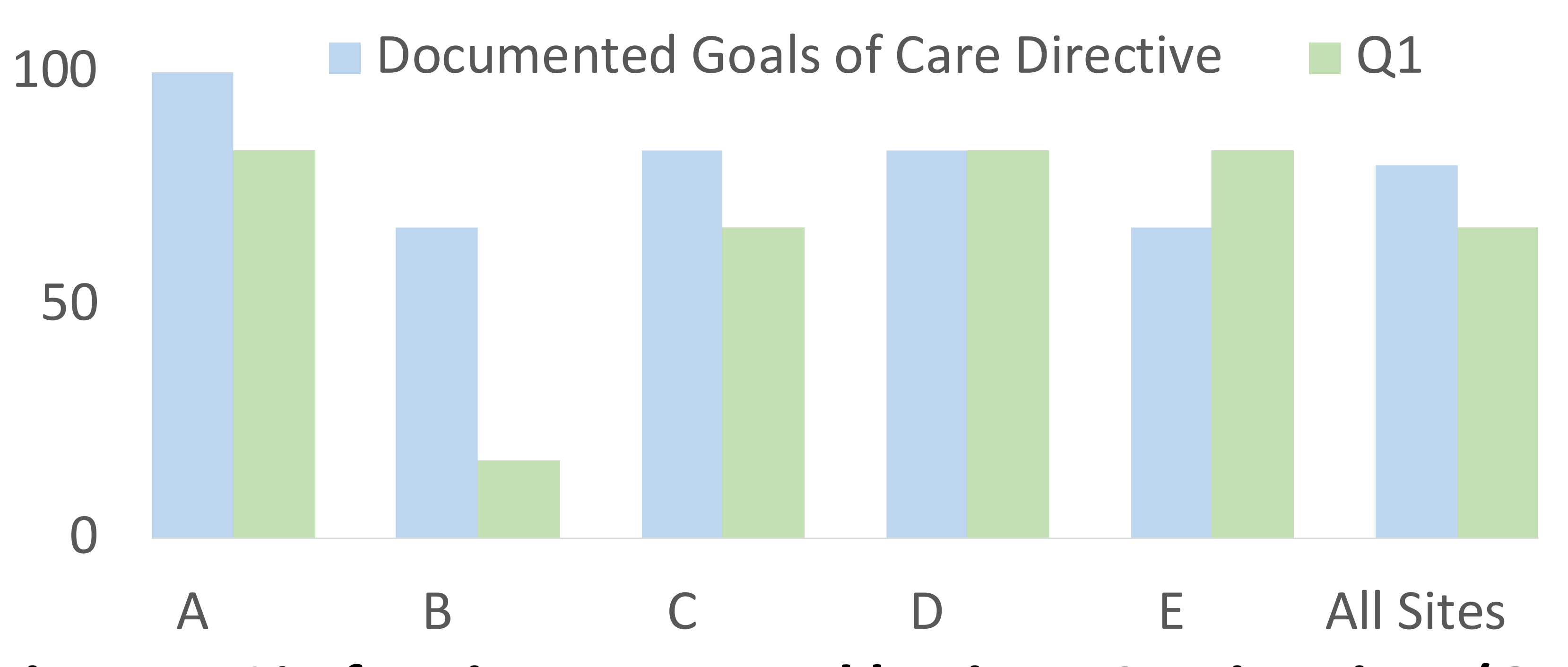

Figure 4. \% of patients reported having ACP Directives (Q1) compared to Goals of Care Directive documented in patient chart.

\section{Conclusion}

- This pre-implementation baseline assessment informs that there is room for improvement in the quality and timing of ACP discussions with renal patients across all BC HAs.

- Evaluation of these components in 1 year post-provincial palliative care initiative implementation will guide further quality improvement initiatives.

\section{Acknowledgements}

We are grateful to the medical teams and patients within the BCHAs and BC Renal for their ongoing support for this project. 